\title{
EL DESARROLLO ESTRATÉGICO DEL TRIBUNAL SUPREMO DE ISRAEL EN RELACIÓN CON LA OCUPACIÓN MILITAR *
}

\author{
Yaniza Giraldo Restrepo
}

THE SUPREME COURT OF STRATEGIC DEVELOPMENT IN CONNECTION WITH ISRAEL MILITARY OCCUPATION

\section{RESUMEN}

ESTE ARTÍCULO ANALIZA LA DISCRECIONALIDAD JUDICIAL QUE TIENE EL TRIBUNAL SUPREMO DE ISRAEL PARA DECIDIR SOBRE TEMAS RELATIVOS A LA OCUPACIÓN MILITAR ISRAELÍ. EL OBJETIVO ES CONOCER CÓMO HA SIDO ESA INTERPRETACIÓN TENIENDO EN CUENTA EL DERECHO CONSTITUCIONAL DE ISRAEL. ENTONCES SE HA VALIDO DE LA HISTORIA, LA JURISPRUDENCIA, LOS VALORES DEL ESTADO Y LA MORAL, OFRECIENDO UNA VISIÓN INTEGRAL DEL DERECHO CONSTITUCIONAL. SE ESTUdiARÁ EL PAPEL QUE JUEGA LA CULTURA DENTRO DEL PROGRESO CONSTITUCIONAL TOMANDO COMO REFERENCIA LA TEORÍA DE LA LEGITIMIDAD EN LA ARGUMENTACIÓN Y HACIENDO REFERENCIA A PETER HÄBERLE SOBRE LAS CONSTITUCIONES, SUS VALORES CONSTITUCIONALES COMO REFLEJO DEL DESARROLLO CULTURAL DEL Estado. Se ANALIZARÁ EL USO DE LAS DIRECTRICES POLÍTICAS Y EL CONFLICTO QUE SURGE ENTRE LA LEY Y LA POLÍTICA, TOMADO COMO UNA ESTRATEGIA POR PARTE DEL TRIBUNAL SUPREMO DE ISRAEL PARA JUSTIFICAR LAS ACCIONES DENTRO DE LOS TERRITORIOS PALESTINOS OCUPADOS. ENTONCES, SE ANALIZA EL PAPEL QUE TIENE ÉSTE EN SU ACTIVIDAD CREATIVA, QUE NO SE LIMITA SOLO AL ANÁLISIS DE LO QUE DICE LA LEY SINO A LAS POSIBLES SOLUCIONES QUE PUEDEN DERIVARSE DE LA MISMA.

\section{PALABRAS CLAVE}

DesarRollo estratégIco; Tribunal Supremo; ocupación MILITAR; PALESTINA.

\section{ABSTRACT}

THIS ARTICLE ANALYZES THE JUDICIAL DISCRETION THAT HAS THE ISRAELI SUPREME COURT TO DECIDE ON ISSUES RELATING TO THE ISRAELI MILITARY OCCUPATION. THE GOAL IS TO UNDERSTAND HOW THIS INTERPRETATION HAS BEEN GIVEN THE CONSTITUTIONAL RIGHT OF ISRAEL. SO HAS USED HISTORY, JURISPRUDENCE, GOVERNMENT SECURITIES AND MORALS, OFFERING A COMPREHENSIVE VIEW OF CONSTITUTIONAL LAW. THE ROLE OF CULTURE WITHIN THE CONSTITUTIONAL PROGRESS BY REFERENCE TO THE THEORY OF LEGITIMACY IN ARGUMENTATION AND REFERRING TO PETER HÄBERLE ON CONSTITUTIONS, CONSTITUTIONAL VALUES REFLECTING THE CULTURAL DEVELOPMENT OF THE STATE WILL BE CONSIDERED. USE OF THE POLICY GUIDELINES AND CONFLICT THAT ARISES BETWEEN LAW AND POLITICS, TAKEN AS A STRATEGY BY THE SUPREME COURT OF ISRAEL TO JUSTIFY THE ACTIONS IN THE OCCUPIED PALESTINIAN TERRITORIES WILL BE ANALYZED. THEN THE PAPER HAVING IT IN HIS CREATIVE ACTIVITY NOT ONLY LIMITED TO THE ANALYSIS OF WHAT THE LAW SAYS BUT POSSIBLE SOLUTIONS THAT MAY ARISE FROM SUCH ANALYZES.

\section{KEYWORDS}

STRATEGIC DEVELOPMENT; SUPREME COURT, MILITARY ocCupATION, PALESTINE.

\section{INTRODUCCIÓN}

El presente trabajo intenta mostrar que la teoría de la interpretación constitucional ha sido vista como ciencia cultural, la cual intenta dar explicación de cómo analizar 
una norma o constitución, porque aunque estas expresen las mismas palabras no siempre van a ser interpretadas igualmente y no tienen el mismo significado. Esto viene determinado por las diferentes épocas en las que haya sido promulgada la Constitución y de acuerdo a su lugar de aplicación (HÄBERLE, 2003, p. XXVI). La interpretación puede variar considerablemente cuando se presenta en situaciones de guerra y conflictos armados nacionales e internacionales. Ahora bien, aunque en una democracia es esencial tener en cuenta la Dignidad Humana como máxima expresión del Estado, hay sociedades que han sido denominadas abiertas y en las cuáles esta no es una regla predominante (HÄBERLE, 2003, p. XXVII). Entonces, hacen una adaptación del derecho a la Dignidad Humana correspondiente con el momento constitucional que está viviendo la sociedad y de acuerdo a las necesidades del Estado; esto sin duda es una interpretación cambiante y utilitarista.

En la interpretación de la Constitución existe un elemento temporal y otro espacial. El primero responde a la tradición histórica, como también a lo estipulado en el proceso constituyente. Así, éste tiene en cuenta todas las experiencias propias del Estado para darle una identidad a las instituciones creadas. En relación con el espacial, se refiere a los diferentes momentos culturales que tiene una sociedad, y sirven para la identificación estatal que marca tendencias dentro del mismo. Este proceso cultural tiene en cuenta no solo la tradición histórica de las sociedades sino también el tiempo que se desarrollan. Puede usarse como punto de referencia para otros Estados en su proceso de desarrollo constitucional, tomando experiencias ajenas y añadiendo otros comportamientos constitucionales propios que en última, pueden aportar avances significativos al Estado constitucional. El objetivo de este artículo es analizar los efectos que ha tenido la aplicación del derecho israelí por parte del Tribunal Supremo, como forma de resolver los conflictos que se presentan a causa de la ocupación en los Territorios Ocupados.

La hipótesis es que la doctrina del Tribunal Supremo de Israel en sus sentencias ha legalizado la ocupación, interpretando las normas del derecho internacional e israelí, justificando las acciones del gobierno militar y la situación de ilegalidad del Estado de Israel. Éste ha traspasado los límites de la legalidad, de la efectividad normativa de acuerdo a cada caso, tomando decisiones arbitrarias y justificadas siempre desde la ley (AGAMBEN, 1998, p. 19). Las leyes aplicadas no han sido firmes y han perdido validez al no ser utilizadas de manera general entre la población de los Territorios ocupados, haciendo una distinción entre la población palestina e israelí, beneficiando en la mayoría de los casos al poder ocupante; no hace distinción entre lo violento y lo legal (AGAMBEN, 1998, p. 22). La doctrina del TSI ha cambiado la teoría del derecho respecto a las leyes internacionales aplicadas en situaciones de ocupación, generando una aceptación y legitimación no solo nacional sino internacional frente a las acciones militares en la región palestina, y una normalización que hace aún más difícil la autodeterminación del pueblo palestino y la creación de un Estado. El problema 
que presenta esta discrecionalidad judicial es que con la idea de la seguridad jurídica y la confianza en la labor del intérprete judicial, se pueden dar razones y justificaciones, aunque éstas no se ajusten a la realidad y la necesidad social (HABA, 1989, p. 44). En la primera parte se analizará el papel desempeñado por el Tribunal Supremo de Israel en relación con la ocupación militar en Palestina, la aplicación de las Leyes Básicas de Israel que hacen las veces de una Constitución Política. En la segunda parte se explica el activismo judicial del Tribunal Supremo de Israel y los efectos que esto ha tenido en los Territorios Ocupados, como parte de su función principal la de resolver los problema derivados de la ocupación. En la tercera parte se explica cómo el Tribunal Supremo de Israel ha reforzado su papel de intérprete constitucional, frente a las acciones del gobierno militar establecido en los Territorios Ocupados.

\section{El Tribunal Supremo de ISRAEl Y los TerRitorios ocupados}

Para empezar hablar del papel que ha desempeñado el Tribunal Supremo de Israel en los territorios ocupados (en adelante, TSI) es preciso anotar que el Estado de Israel no tiene una Constitución escrita, ${ }^{1}$ por tanto, la interpretación constitucional se toma de las Leyes Básicas. Posterior a la creación del Estado de Israel en el año 1948 y al no llegar a un consenso sobre la creación de una Constitución en el sentido "rígido y formal" (SEGAL, 1992, p. 2), se propuso la promulgación por capítulos de los derechos humanos por capítulos tal como se había hecho hasta entonces (BARAK-EREZ, 1994-1995, p. 323-326). ${ }^{2}$ Las primeras dos leyes Básicas que se crearon en Israel para proteger los derechos fundamentales fueron: Ley Básica: "Dignidad Humana y Libertad"; 3 Ley Básica "Libertad de Ocupación". 4 Esto marca un antes y un después en el sistema constitucional de Israel y por eso se dice que desde 1992 "empezó una nueva era constitucional” (BARAK-EREZ, 1994-1995, p. 323).

Al no existir una constitución en Israel no hay un Tribunal Constitucional, entonces elTSI ha tomado como función propia la interpretación de las Leyes Básicas, cuando tiene que decidir sobre las violaciones a los derechos humanos por parte del gobierno militar de Israel, establecido en los Territorios Ocupados por Israel en 1967 (en adelante TO). ${ }^{5}$ Este gobierno militar pertenece a las Fuerzas de Defensa de Israel, y está encargado de mantener el orden y la seguridad de la región ocupada. Por esto, el TSI conoce algunas demandas presentadas por abusos de autoridad y violación de los derechos humanos a la población palestina, porque este gobierno militar hace del Estado de Israel. De conformidad con el derecho internacional cuando hay una ocupación de un territorio, es posible establecer una autoridad militar de la fuerza ocupante, que será la encargada de mantener el orden en la región (ROBERTS, p. 58-62).

Aunque el Estado de Israel es un Estado democrático, que busca mantener el Estado de Derecho, y la protección de los derechos humanos, las decisiones tomadas dentro de los TO son diferentes a las aplicadas dentro de su Estado; en este sentido, se observa 
una ambivalencia porque esa protección jurídica no siempre será la misma cuando se trata de dirimir los conflictos que se presentan por causa de la ocupación militar En este sentido, el Juez Aharon Barak (2008, p. 1) determina que la función principal de un Tribunal Constitucional en democracia no es solamente la de corregir los errores cometidos en las sentencias, sino también, la importancia de "cerrar la brecha entre el derecho y la sociedad y proteger a la democracia”.

La competencia del TSI para conocer las demandas por violaciones a los derechos humanos de la población palestina a causa de las acciones del gobierno militar, deriva de la capacidad que tiene este de conocer y juzgar a los funcionarios públicos dentro o fuera de Israel; por eso, puede decidir sobre la legalidad de las acciones militares dentro de los TO, confirmando o anulando las mismas. Una de las principales funciones del TSI es la de resolver los problemas que surjan entre la población y las autoridades del Estado, teniendo como objetivo principal asegurar el "Estado de Derecho" (NETANYAHU, 1993, p. 2; STRASCHNOV, 1998-1999). ${ }^{6}$ Por otra parte, crea políticas de protección de los derechos humanos y ejerce el papel de Tribunal Constitucional en ausencia de una Constitución escrita, lo que ha generado críticas por considerar que se excede en sus funciones; esta actividad ha generado propuestas para la separación de sus actividades constitucionales (AHARON, 2003, p. 15) ${ }^{7}$. Ahora bien, si crearan un Tribunal Constitucional podría haber mayor independencia en las decisiones que toman en los temas relacionados con los TO o del mismo gobierno, lo que contribuiría a generar mayor seguridad jurídica en el Estado.

La metodología empleada por el TSI para la interpretación constitucional tiene en cuenta los valores democráticos del Estado, éstos otorgan jurisdicción al mismo para decidir sobre los conflictos que puedan presentarse entre el Estado y las personas. Los valores democráticos sin duda constituyen la base fundamental para la garantía de la justicia (GOMES, 2004, p. 39). Ahora bien, éste recurre a unos valores constitucionales que no están propiamente definidos dentro del Estado pero provienen de unos criterios de interpretación de la ley que no pueden ser tenidos en cuenta literalmente como una interpretación constitucional (DÍAZ, 1997, p. 299).

Como ha destacado Díaz (1997, p. 300), la función principal de la interpretación constitucional tiene en cuenta las disposiciones constitucionales vistas desde una óptica diferente dependiendo del intérprete, por esto, el juez y el legislador pueden llegar a soluciones distintas. Entonces, la función de las normas constitucionales es aclarar la zona de incertidumbre que se puede presentar y constituye la función principal del intérprete constitucional. Que no tiene la "única respuesta correcta" sino que interpretará cuales son las posibles interpretaciones que pueden resultar inadmisibles, es decir "campo de licitud” a otros operadores jurídicos. Así las cosas, el TSI conoce las demandas presentadas por la población residente en los TO sin oposición hasta la fecha del gobierno israelí. La función desempeñada por el TSI no ha tenido precedentes en el derecho internacional y esto ha modificado la teoría del derecho 
internacional, dadas las características particulares de ocupación prolongada (EDELMAN, 1994, p. 32).

La tradición jurídica del TSI ha sido recibir las demandas presentadas por la población de Cisjordania y la Franja de Gaza desde su ocupación (1967), no obstante, solo a partir de la promulgación de la Leyes Básicas reafirmó su competencia en relación con la protección de los derechos humanos en los TO (STRASCHNOV, 1998-1999, p. 532-533; HOLZMAN-GAZIT, 2007, p. 35). En la jurisprudencia del TSI se puede observar la influencia del derecho internacional y los tratados ratificados por el Estado, que han servido para dirimir casos difíciles y en ocasiones han beneficiado a la población palestina (KRETZMER, 1996, p. 97)..$^{8}$

El activismo del TSI ha sido constante a causa del incumplimiento de las obligaciones del gobierno militar en los TO, el exceso de poderes y la suspensión de los derechos fundamentales dentro de los TO por el Estado de Israel y sus políticas gubernamentales, donde en la mayoría de casos las decisiones no benefician a la población palestina (MCEWEN, 1986). En esta misma línea, el TSI en ocasiones ha tenido que decidir sobre la legalidad de las funciones desempeñadas por el gobierno militar en los TO, conocer sus excesos en sus poderes discrecionales, resolver las demandas presentadas por los ciudadanos palestinos contra las autoridades públicas incluidas las fuerzas de seguridad israelí; las sentencias del TSI constituyen la última estancia. ${ }^{9}$

La función que desempeña el TSI como garante de los derechos fundamentales no solo en el Estado sino fuera del mismo, no es nada contradictorio teniendo en cuenta que el denominado Estado Constitucional desde la teoría de Peter Häberle (2003, p. XXXI), tiene como referencia no solo aquellos Estados con una Constitución escrita, sino que destaca la importancia de proteger los derechos fundamentales inderogables e intransferibles, la separación de poderes, y la ley como el resultado de la expresión popular, así no exista una referencia escrita. No importa el tipo de Constitución existente o la denominación que goce, porque en el constitucionalismo contemporáneo se tiene en cuenta la democracia.

El papel que ha jugado el TSI es determinante para la protección de los derechos fundamentales de los palestinos, porque a pesar de que en el Estado no existe una Constitución escrita, su función ha sido la de un Tribunal Constitucional. Su actividad es cuestionada en Israel porque interviene en temas tan controvertidos como los asentamientos israelíes, los excesos de las Fuerzas de Defensa israelí (en adelante FDI), las ejecuciones extrajudiciales. Sin embargo, en ocasiones el TSI ha hecho caso omiso a las presiones de los diferentes grupos políticos y por el contrario, ha realizado una interpretación evolutiva, tomando no solo lo dispuesto en las Leyes Básicas del Estado, sino haciendo una aproximación a la realidad social palestina. Esto ha sido denominado como "modelo axiológico de la constitución concebida como normas" (POZZOLO, 1998), que utiliza el juez en la interpretación y argumentación, como elemento central del razonamiento para poder decidir. Entonces, no es posible conocer 
el sentido de las "Leyes Básicas" si no se tiene en cuenta la justificación de la decisión que realiza el TSI de conformidad con ese modelo axiológico. En la interpretación que hace el TSI del derecho Israelí para un caso concreto en Palestina, y aunque estas representan un límite en el ordenamiento jurídico, le han permitido dar una sustentación jurídico-moral en los TO. No obstante, se trata de una interpretación no siempre sometida al Derecho internacional, sino principalmente al Derecho israelí, lo que da lugar a arbitrariedad en la aplicación normativa en los $\mathrm{TO}^{10}$ (POZZOLO, 1998, p. 342).

Así las cosas, para evitar problemas de arbitrariedad en la decisión judicial, derivada de la interpretación de las "Leyes Básicas", es necesario tener en cuenta el concepto de "regla de reconocimiento" expuesto por Hart, donde la regla de reconocimiento sí es una norma positiva, pero no es una norma divulgada, sino que es una regla social convencional aceptada implícitamente por los jueces (HART, 2012, p. 97). Para que exista una regla de reconocimiento en un sistema jurídico se requiere que haya un acuerdo general en comunidad acerca de su contenido, aunque no quiere decir que no puedan existir diferencias en situaciones específicas; donde los principios que actúan como normas morales positivas no dan explicación de los tipos normativos, sino el reconocimiento de algunos de ellos. Estos principios son esenciales para justificar cómo un tribunal pasa de una disertación jurídica a la moral, y son la base para la sustentación de las decisiones jurídicas (POZZOLO, 1998, p. 343). Según Pozzolo,

Es preciso, pues, modificar la regla de reconocimiento y aceptar el hecho de que, en ciertos casos, y dado que los principios son jurídicos, el derecho es considerado válido no en razón de un test de pedigree sino en razón de un test moral, el cual, por definición, no se basa en la génesis de los principios sino en su contenido. (1998, p. 343)

Se entiende que la "regla de reconocimiento" tendría que tener en cuenta los principios y valores que legitiman no solamente el ordenamiento jurídico interno de Israel, sino los principios y valores de la comunidad a la que va dirigida las leyes y también las normas internacionales de ius cogens. Dada la situación especial de ocupación y las actividades militares que ahí se desarrollan. Los principios tendrían entonces la función de servir de parámetros de justificación argumentativa para la decisión judicial, no porque estén destinados a favorecer o asegurar una situación de carácter económico, social o política, sino que responden a una exigencia de la justicia, la legalidad o todo aquello relacionado con la moral (DWORKIN, 2012, p. 72).

En este sentido, Shapiro contrario a la teoría de Dworkin, establece que los jueces en realidad no están planteando desacuerdos teóricos, sino que están buscando una justificación para ocultar la verdadera razón y es la de "legislative nature". Esto se da precisamente según Shapiro, porque en un sistema democrático donde existe un 
sistema de separación de poderes los jueces solo están obligados aplicar la ley, y resulta peligroso que acepten la discrecionalidad y la modificación de las leyes a través de sus sentencias. Esta es la forma en que por ejemplo, el TSI, podría conservar su legitimidad reconociendo la ley e interpretándola, más no modificando la misma (2007, p. 33). Ahora bien, esta discrecionalidad muestra la potestad legislativa que está ejecutando el TSI, la discreción judicial cumple la norma establecida en las Leyes Básicas, pero esta actuación en ocasiones es arbitraria porque se aplica a una comunidad, en este caso la palestina, que es diferente y no ha tenido participación en la creación de las mismas.

Entonces, en la decisión judicial delTSI en relación con los TO será necesario tener en cuenta los principios jurídicos, porque éstos permiten reducir la arbitrariedad y conducen el acercamiento a la "justicia". El problema de la interpretación de las Leyes Básicas del Estado de Israel, bajo la teoría moral (DWORKIN, 2005), es que hace injustas las decisiones judiciales tomadas en relación con los TO, porque estas responden a los valores judíos del Estado de Israel, son leyes aprobadas para otra comunidad jurídica, con otras condiciones sociales muy diferentes a las de la población de los TO; por ello, es necesaria la concreción conceptual del TSI que asegure la justificación argumentativa. Como ejemplo de las decisiones injustas y tomadas en razón de la moral de las Leyes Básicas, tenemos la sentencia del TSI 10356/02, Hass v IDF Commander in West Bank, donde se confirma la decisión del comandante de las FDI en Cisjordania de ampliar la ruta de los peregrinos que conduce hacia la cueva Machpela, permitiendo la expropiación de terrenos de propiedad privada y demoliendo algunos edificios. Los demandantes solicitaron la revisión de esta orden del comandante de las FDI ${ }^{11}$ por considerarla ilegal y desproporcionada a la vista de la finalidad para la cual fue hecha. Se fundamentan en el daño a la propiedad privada y al patrimonio arqueológico.

Por otra parte, sostienen los demandantes que en realidad las motivaciones fueron políticas porque se buscaba la continuidad territorial entre Kiryat Arba ${ }^{12}$ y la cueva de Machpela, que en el futuro permitirá la expansión de los asentamientos israelíes. Se alegó que no había una conexión entre los ataques que se produjeron en la zona y las medidas previstas, entre las cuales estaban la destrucción de viviendas, pues esta zona había sido declarada previamente zona militar, desalojando a las personas que vivían en ella. ${ }^{13} \mathrm{~A}$ su vez, también argumentaron que la expropiación de la tierra y la demolición de los edificios que se rigen por esta orden es contraria al derecho internacional, que requiere del comandante del área ejercer su autoridad para garantizar el orden y la seguridad en la zona ocupada, en el virtud del art. 43 de la Convención de La Haya de 1907 y al art. 53 del Convenio de Ginebra, relativo a la protección debida a las personas civiles en tiempo de guerra de 1949. En éstas se prohíbe la destrucción de las viviendas de los civiles en una zona ocupada a no ser que esto se haga como una acción esencial y necesaria para los procedimientos militares. La orden de movilización también es contraria a la ley internacional que regula la conservación 
de los bienes arqueológicos. ${ }^{14}$ Ante estos argumentos el Estado de Israel respondió que el único interés en realidad era el de proteger a la población judía contra los ataques terroristas, cuando estos visitaban la ruta de los peregrinos. Que en un análisis de otras opciones a la destrucción de viviendas, se tomó esta última como la opción más adecuada para la protección de la población civil. Así, la legalidad de esta orden fue analizado dentro del marco del derecho internacional, el derecho local y la legislación israelí de la Ley Básica: Dignidad Humana y Libertad.

ElTSI en el caso de la cueva de Machpela estableció ${ }^{15}$ que los habitantes de la región tienen un derecho constitucional a la libertad de religión y de culto, que aplica tanto para los habitantes árabes y también en el caso de los habitantes judíos que viven allí. Por otra parte, tienen el derecho de libre circulación, a través del cual es posible ejercer el derecho de acceso a los lugares santos. Así las cosas, el derecho de circulación y el acceso a los lugares santos es de carácter constitucional y constituye uno de los derechos humanos básicos. Sin embargo, establece que los derechos de libertad de religión y de culto no son de libertad absoluta, sino relativos, y por eso es necesario hace un equilibrio entre estos y otros derechos y valores que son dignos de protección, incluido el valor de la propiedad privada.

El TSI resuelve que el derecho de una persona a la propiedad no es un derecho absoluto sino relativo porque puede ser vulnerado cuando sea necesario proporcionar el avance de los diferentes derechos constitucionales básicos de los demás. ${ }^{16}$ Concluye pues que la ponderación entre los derechos constitucionales en conflicto no es fácil pues se trata de los derechos de la expresión humana a través de la práctica de creencias y de culto religioso, que entran en conflicto con los derechos y los valores relativos a la tierra y la propiedad, que entran en choque con un valor general de la responsabilidad de proteger la vida humana. Así que la decisión tomada por el comandante del área satisface la prueba de constitucionalidad, porque buscó un equilibrio relativo entre estos derechos constitucionales. ${ }^{17}$

Díaz (1997, p. 304-305), explica que dentro de la interpretación constitucional se puede dar una peculiaridad como es la de la politicidad, que viene a incidir dentro de las disposiciones constitucionales como los sujetos que participan dentro de ese proceso constitucional. Entonces, la interpretación constitucional estará inevitablemente vinculada con los grupos de poder político y social que en ocasiones pueden condicionar las decisiones del Tribunal Constitucional o en el caso de Israel, ir en contraposición frente a la política del mismo Estado. Estos cambios del TSI han sido significativos beneficiando en ocasiones a la población palestina, aunque criticados dentro del Estado de Israel al considerar que asume un papel de creador o legislador. Este activismo judicial ha sido importante dentro de los TO porque representa un límite a las acciones del gobierno militar, sin embargo, no es justo ni suficiente porque al decidir teniendo en cuenta el derecho de Israel, es evidente que no siempre va a beneficiar a la población palestina al hacer de juez y parte dentro del conflicto. 
A continuación se analizarán los efectos de la actividad judicial del TSI y cuáles son las fuentes del derecho que utiliza para la interpretación constitucional.

\section{Activismo Judicial del Tribunal Supremo de Israel}

El TSI ha utilizado la interpretación constitucional como un reflejo de la cultura democrática del Estado, teniendo en cuenta las normas Constitucionales en este caso las Leyes Básicas, para su aplicación en la sociedad y en todas las situaciones de conflicto que se presente, como es el caso de los TO (HÄBERLE, 2003, p. XXXIV). La teoría de Häberle (2003, p. XXXV) habla de una sociedad abierta donde todos los integrantes de la sociedad, agentes del Estado, partidos políticos, y todo aquel que hace parte de la misma debe regirse por la norma suprema incluso si dentro de la interpretación se tienen en cuenta "los efectos jurídicos, políticos y morales de los actos de interpretación".

Se puede decir que el TSI ha creado un nuevo derecho a través del establecimiento de normas jurídicas no aceptadas como una Constitución, que han pasado a ser preceptos jurídicos de obligatorio cumplimiento, como si se hablara de unas normas erga omnes. Para esto, recurre a la interpretación de las Leyes Básicas, los valores democráticos del Estado de Israel, que le permiten dar resolución a problemas y conflictos normativos. Por tanto, no se limita solamente a un proceso deductivo de las normas sino a su adaptación social (DÍAZ, 1997, p. 327-328).

En consecuencia, el TSI ha tenido un papel decisivo en la interpretación Constitucional porque no solamente ha interpretado las Leyes Básicas, sino que también ha afianzado las mismas dentro del sistema israelí como si fueran una Constitución escrita. En las diferentes sentencias ha ido más allá de estas Leyes Básicas, haciendo una adecuación de las mismas a la situación de los TO (HÄBERLE, 2003, p. XXXIX). La actividad judicial delTSI ha sido criticada por los grupos religiosos a causa de las decisiones que ha tomado en materia religiosa y de Estado (SHETREET, 2002-2003, p. 723-724). Se debe tener en cuenta que en Israel no hay una separación de la religión y el Estado, y esto sin duda limita el desarrollo Constitucional y la actividad de los tribunales. El TSI se ha pronunciado en los casos que afectan la seguridad nacional y en ocasiones, ha cuestionado las medidas tomadas por el gobierno y los excesos de los mismos considerando que no se respetó el principio de proporcionalidad. ${ }^{18}$

El proceso Constitucional que ha vivido el Estado y el activismo del TSI se pueden dividir en dos etapas que marcan un antes y un después en la protección de los derechos fundamentales, demostrando un desarrollo que sin duda ha beneficiado a la población de los TO (DOTAN, 2004, p.190), lo que es denominado como "Revolución Constitucional":

a) Desde la creación del Estado de Israel en 1948 hasta 1970 desarrolló una amplia jurisprudencia de revisión judicial donde se estableció los principios de la regla 
del derecho, creando una conciencia de protección de los derechos humanos que servirían posteriormente para promulgar las "Leyes Básicas". Todo este desarrollo se generó al margen de involucrarse en debates políticos o controversias públicas (DOTAN, 2002, p. 93). En este periodo limitó la posibilidad de plantear cuestiones políticas tratando solo temas que estuvieran relacionados entre el individuo y el Estado. Centró su interés en las acciones del Estado lo que evitó que el TSI entrara a dirimir casos de religión y Estado. En este periodo no tomó en cuenta temas jurídicos relacionados con la Política Exterior, acciones militares u otros asuntos que generaran un amplio debate y que consideró inadecuados para una decisión jurídica (BARAK, 2008, p. 104; DOTAN, 2002, p. 94). ElTSI tuvo en cuenta dos conceptos: la justiciabilidad normativa que establece si existen criterios jurídicos para determinar el conocimiento de una disputa y la justiciabilidad institucional que determina si la disputa se resuelve por una Corte Jurisdiccional.

b) Hacia el año 1980 y 1990 (HOLZMAN-GAZIT, 2007, p. 48-49) el TSI tiene mayor interés jurídico por conocer casi todo tipo de casos presentados incluso los de interés individual y que antes eran inadmitidos. Este cambió se dio a partir del caso Ressler donde una cuestión de importancia constitucional y de violación del derecho por parte del gobierno, puede ser presentado por cualquier persona ante el TSI ${ }^{19}$ algo que refleja su innovación judicial y la aplicabilidad del concepto de justiciabilidad que es desarrollado a partir de la fecha.

De manera que el TSI dejó de ser solamente un organismo encargado de resolver las disputas entre los individuos y el Estado, a ser un garante del Estado de Derecho. En la jurisprudencia del TSI se establece que cualquier acción humana es susceptible de ser analizada por medio de una norma legal dejando atrás el concepto de posible vacío legal (DOTAN, 2002, p. 95). Se puede decir que el TSI recurre a la teoría de la interpretación que habla Alexy (2008), para resolver los diferentes conflictos jurídicos que en principio parecieran no tener solución, entonces, crea un nuevo concepto o hace una ponderación de normas para dar una solución; entonces, las obligaciones legales surgen de la misma ley.

En este sentido, el TSI interpreta y desarrolla la teoría del derecho y el papel de los tribunales nacionales como creadores o transformadores, tomando como fuente el derecho internacional. En los TO ha decidido en temas de violación de los derechos humanos haciendo una ponderación en la aplicación del Derecho Internacional Humanitario y el Derecho Internacional de los Derechos Humanos (COHEN, 1985-1986). Esto se da porque en la actualidad el juez, y en particular el juez constitucional, gozan de gran discrecionalidad. Así las cosas, lo que puede suceder es que el juez interprete teniendo en cuenta su ideología que puede variar de un caso a otro, sin parámetros que se sostengan como en el Stare decisis. Esto muestra cómo el TSI toma una política 
propia sin tener en cuenta el papel de los valores y principios ligados a las garantías y derechos fundamentales. Dworkin establece que la decisión judicial se basa en las instituciones jurídicas y la ley, que será aquella decidida anteriormente y por ello, no es posible en un caso difícil dejar libertad al juez para decidir pues no está facultado para crear normas. Entonces, el juez debe buscar principios y criterios claros, sustentados en las teorías que conceptualicen y justifiquen su decisión. (1986, p. 13-15). Hay que tener en cuenta que las acciones del TSI responden a la necesidad de dar una respuesta a las demandas presentadas por la población palestina frente a los abusos de la autoridad militar.

Dworkin en su debate con Hart sustenta que el juez no debería tener discrecionalidad judicial en el momento de la decisión porque en todo caso debe acudir a los principios como parámetro para la interpretación normativa. La directriz política según Dworkin es "un tipo de estándar que propone un objetivo que ha de ser alcanzado", donde se busca mejorar en algunos aspectos económicos, políticos o sociales de la comunidad. El principio es un estándar que ha de ser observado, pero no porque intente beneficiar o asegurar que se cumpla una situación económica, política o social sino porque así lo exige la justicia o equidad u otra exigencia de la moral (2012, p. 72).

El TSI ha utilizado el concepto de discrecionalidad judicial para interpretar los casos relacionados con las acciones militares y los servicios de seguridad dentro de los TO, ${ }^{20}$ haciendo uso de la opción valorativa y tomando como punto de referencia las Leyes Básicas. Sin lugar a duda, este razonamiento jurídico puede llegar a ser peligroso porque el juez no solo cuenta con la limitación del método empleado y las reglas, sino que tiene en cuenta opciones valorativas en cada caso (DÍAZ, 1997, p. 329-330). La jurisprudencia del TSI se centró en los principios del derecho, la moral, la sociedad, los valores del sistema legal, ganando mayor protagonismo dentro de la sociedad israelí. Todo esto sin duda responde al papel del juez dentro de una sociedad y es la de hacer un análisis de todos los principios y normas, para determinar cuál será la que mejor se adapte a una decisión judicial justa.

El balance establecido por el TSI entre la política y la democracia ha sido denominado como "judicial activism” (NAVOT, 2007, p. 62), ampliamente criticado por ser visto como un activismo que genera restricciones, porque otorga al TSI una posición dominante y superior para dirimir cuestiones Constitucionales y vacías que no están claras dentro de las Leyes Básicas (GOLDSTEIN, 1999, p. 62). Este concepto es señalado por Dworkin como "poder creador del juez" donde el juez introduce nuevos derechos para ser aplicados retroactivamente (2012, p. 152), y a pesar de que éste no debe legislar cuando lo haga se pondrá en la posición del legislador y resolverá el caso como lo haría el mismo (DWORKIN, 2012, p. 147). Entonces, el autor llega a la conclusión que en algunos casos nuevos que no cuentan con un precedente judicial, aunque se interpreten las normas no se podrá dar respuesta al problema, siendo inevitable el poder del juez de interpretar y transformar las mismas; esto no 
equivale a una decisión sin límites y debe tener en cuenta la racionalidad, la justicia y la eficacia de las normas. (DWORKIN, 2012, p. 80-86).

Ahora bien, cuando se va a decidir en un caso, es necesario tener en cuenta argumentos basados en principios jurídicos, que sirven como estándares diferentes de las normas, donde los principios vienen a tener una posición importante en los argumentos presentados por el juez, cuando fundamenta ciertos derechos y obligaciones jurídicas. En la interpretación judicial cuando los tribunales actúan en algunos casos como delegados del poder legislativo, es inevitable que utilicen argumentos políticos.

En el "Imperio de la Justicia", Dworkin aclara aún más el problema que se presenta en la interpretación de los tribunales y la decisión en los casos difíciles, argumentando que es una tarea básicamente interpretativa, y determina una teoría del Derecho como integridad. Ésta teoría establece que los jueces deben seguir interpretando que el derecho está conformado por un conjunto de principios rectores basados en la justicia, equidad y debido proceso, siendo su función la aplicación de los mismos (2005, p. 164-165). Entonces, esa es la función que supone la teoría de la integridad del derecho como un ideal político y los jueces tomaran sus decisiones teniendo en cuenta el derecho consuetudinario basados en los principios (DWORKIN, 2005, p. 176). En consecuencia, si no se atiende la teoría del derecho como integridad, la decisión del juez puede ser ambigua y es muy arriesgado pensar que la respuesta estará en la moralidad política de una comunidad, impositiva de sus valores a otra que no comparte los mismos y qué tan solo el papel del juez es hacer valer sus derechos y reconocer e interpretar los mismos (de su misma comunidad y no de otra). Entonces, los tribunales deben tener en cuenta una teoría general del actual derecho, que refleje principios coherentes de equidad política, justicia sustantiva y debido proceso (DWORKIN, 2005, p. 284).

Desde otras posturas teóricas, lo mejor sería analizar la regla de reconocimiento que sirve tanto para la adopción y creación de normas en una comunidad, y además, posee un valor interpretativo. En este sentido, el TSI ha conocido diferentes casos donde no existe un precedente judicial, de manera que hace una interpretación de la situación, adoptando con ello una mayor intervención judicial en las decisiones tomadas por el gobierno, el Knesset (parlamento israelí) y la administración, en el caso del conflicto palestino-israelí (NETANYAHU, 1993, p. 21-23). El Gobierno de Israel no siempre ha podido hacer frente a los problemas que se plantean en los TO, por esto, a la población no le queda otra alternativa que confiar al TSI la resolución de los casos que han permanecido sin resolver por parte de las autoridades políticas.

El TSI ha determinado que los órganos del Estado están obligados por ley como cualquier otro ciudadano en el Estado, por eso, las acciones militares en los TO pueden ser juzgadas como parte de la regla del derecho, y principio fundamental del Estado. Este principio ha sido desarrollado a través de su jurisprudencia, creando una doctrina que sirve como fundamento para la revisión judicial, donde tiene en cuenta 
las funciones del gobierno y la prohibición de excederse en los poderes otorgados a éste por el poder legislativo (DOTAN, 2002, p. 89). La tendencia jurídica es que los tribunales internacionales toman como referencia los principios constitucionales para la interpretación y creación de nuevas normas, partiendo de los valores democráticos establecidos en cada Estado. El riesgo de esto es la subjetividad de los mismos a la hora de crear las técnicas de interpretación y producción de disposiciones que no están contenidas en la Carta Constitucional; lo que puede llegar a generar una arbitrariedad interpretativa. La oposición del gobierno frente al activismo judicial del TSI es porque considera que no es una actividad exclusiva del mismo y por tanto, va en detrimento del poder Ejecutivo (BRUCE, 2010, p. 192). Este rechazo del gobierno puede constituir una amenaza a la independencia judicial delTSI, porque aunque éste tiene una tendencia internacional hacia la protección de los derechos humanos, en Israel no existe una Constitución escrita o unas leyes supraconstitucionales que determinen la competencia del mismo, como garante de los derechos fundamentales (BARZILAI, 1999, p. 16-17; NETANYAHU, 1993, p. 13-15).

En una democracia como la israelí donde no existe una legislación que regule el papel del TSI y su relación judicial con otras ramas del poder público, cuya estructura gubernamental está en constante cambio, hace mucho más necesaria la creación de una Constitución; esta permitiría definir su competencia y la regulación de los valores básicos de la sociedad, teniendo en cuenta la pluralidad por encima de cualquier interés particular (GORDON, 1998). En este sentido, Gavison (2002, p. 19), sostiene que es importante para Israel una Constitución que permita el entendimiento de un marco político común, en el que cada grupo social pueda actuar a pesar de sus desacuerdos y conflictos de intereses. Porque la sociedad israelí está fragmentada y en un conflicto permanente, puede parecer poco necesario el debate constitucional, aunque en realidad podría ser el mejor momento para el mismo. Sería entonces beneficiosa la creación de un proyecto Constitucional que pueda ofrecer la oportunidad para un debate nacional.

No obstante, ante la ausencia de un Tribunal Constitucional, la jurisprudencia del TSI puede servir para legitimar su competencia, porque se ha fundamentado principalmente en el desarrollo y protección de los derechos humanos, complementado con la doctrina internacional. A su vez, como garante del Estado democrático (PEABODY; WELLS, 2010, p. 193), siendo este un principio empleado por el TSI como base para el reconocimiento de los derechos fundamentales (KRETZMER, 2005, p. 44). Ahora bien, el TSI se enfrenta a varios dilemas a la hora de dictar las sentencias. Primero, el conocimiento de diferentes casos relacionados con la política, religión y seguridad nacional, temas muy sensibles dentro de la sociedad israelí. Segundo, tiene que hacer un balance entre la seguridad del Estado al cual pertenece y por otro lado, proteger los derechos humanos en tiempos de crisis (SHETREET, 2002-2003, p. 715). Esto no debería suponer un problema si la doctrina del TSI se basara en lo dispuesto por las leyes 
de ocupación beligerante, los valores y principios consagrados por la Comunidad Internacional y las Leyes Básicas si estas se aplican sin distinción de credo, teniendo en cuenta que Israel se denomina un Estado judío y Democrático. La posición que sostiene el autor Harold Hongju (2002, p. 23), es que no solo se debe tener en cuenta lo que prohíben o permiten las leyes sino la esencia para la cual han sido creadas las mismas.

Así las cosas, este margen de discrecionalidad que tiene el TSI puede ser riesgoso porque se podría establecer dentro del Estado, decisiones contrarias a los derechos humanos y en particular, el derecho que tienen los pueblos de autodeterminación. Es decir, que sus preferencias y sensibilidades morales son el reflejo de lo que piensa una sociedad en particular, cuando en un momento determinado, en esa decisión está necesariamente comprometida una comunidad disímil (KOSKENNIEMI, 2008, p. 13). Al ejercer su jurisdicción dentro de los TO no solamente logra normalizar esta situación sino que también, le otorga cierto grado de superioridad para conducir y regularizar algo inaceptable como es la ocupación israelí. Koskenniemi señala que esto puede llegar a poner en peligro la importancia de la moral y la política de la realidad de la ocupación, como la necesidad de poner fin a la misma (KOSKENNIEMI, 2008, p. 13).

Es precisamente lo que hace el TSI en su jurisprudencia, toma decisiones respecto a los conflictos y abusos que se presentan en los TO entre la población y el gobierno militar, pero sin entrar a discutir o criticar el fondo del asunto: la ocupación. Esto es algo que no cuestiona y lo justifica por su posición de Tribunal del Estado que lucha contra el terrorismo. La posición del TSI no es fácil, porque hace de juez y parte dentro del conflicto que se presenta a causa de la ocupación de Israel, no obstante, su activismo judicial puede ser un mecanismo importante en la protección de los derechos humanos de los palestinos. A su vez, al ser un Tribunal de un Estado democrático, es su función la de controlar las acciones militares y los abusos que se puedan cometer a causa de la ocupación militar. El TSI decide sobre temas políticos, religiosos y de seguridad, por tanto, debe tener en cuenta no solo los intereses de su Estado, sino la obligación del mismo de proteger los derechos humanos en cualquier situación, en este caso, las derivadas de la ocupación militar dentro de Palestina.

De ahí la importancia de la teoría de la interpretación (SCHAUER, 1992, p. 739741), que permite identificar la naturaleza del derecho y la identificación de las disposiciones imperativas que corresponden a la llamada regla de reconocimiento. El juez toma el caso, analiza las distintas premisas y las interpreta con las normas correspondientes (MORESO, 1997, p. 190). El TSI ha utilizado la teoría moral (moral theory) en su reconocimiento y razonabilidad normativa, sin embargo, no ha resuelto el dilema que se presenta cuando ésta plantea problemas interpretativos porque las normas constitucionales apuntan en una dirección y la teoría moral en otra (SCHAUER, 1992, p. 741).

Es decir, la interpretación es dependiente y no antecedente a la selección de un método de interpretación. Se debe tener en cuenta que hay fuentes de interpretación que no necesariamente son las leyes constitucionales sino que existen otros métodos 
de reconocimiento de las normas y no necesariamente son secundarios sino inherentes a la misma (SCHAUER, 1992, p. 742). Para hacer uso de la teoría de la interpretación y escoger cuál método será el empleado, es necesario identificar el caso, saber si la interpretación puede ser útil al mismo y determinar en principio cuales serían las teorías a utilizar. Ésta teoría constitucional sirve al juez constitucional para decidir las fuentes adecuadas en la interpretación constitucional, como también, en el reconocimiento de los casos difíciles (SCHAUER, 1992, p. 746).

El TSI toma como referencia no solo las leyes escritas sino aquellas que reflejan los valores judíos de Israel, es decir, hace una ponderación de valores denominada en lengua inglesa como balancing, crea un contrapeso de valores e intereses constitucionales decidiendo cuáles serán los derechos que prevalecerán (DÍAZ, 1997, p. 233-234). Según Gavison (2006, p. 211), el papel de los tribunales es la protección de los derechos humanos como base fundamental de su mandato, contra los intereses de la mayoría incluso por encima de cualquier credo. Ahora bien, esa protección de los derechos constitucionales cuando no están claramente incorporados en la ley y su ámbito de aplicación es controvertida, puede ser visto como una adecuación de los tribunales y la aplicación de sus valores sobre los de la mayoría; los tribunales constitucionales son intérpretes pero no creadores de la Constitución (ALBERT, 1969, p. 1263).

La doctrina creada por el TSI en relación con la protección de los derechos civiles y políticos ha cambiado con los años, partiendo de un limitado alcance judicial a una mayor implicación del mismo. En algunas ocasiones ha beneficiado el Estado de Derecho pero en otros ha generado dudas acerca de su legitimidad para intervenir en todo tipo de casos (GAVISON, 2005, p. 18). Se puede señalar que el activismo del TSI se debe a la falta de resolución del gobierno y las evasivas a los problemas derivados de la ocupación, evitando tomar decisiones que son de carácter político o económico (SHETREET, 1990, p. 405-406). El Knesset tiene la posibilidad de limitar el alcance del TSI pero hasta la fecha no lo ha hecho, y puede ser porque las decisiones judiciales de éste no limitan la libertad del gobierno o del poder legislativo (GAVISON, 2005, p. 27).

Por consiguiente, se puede decir que la jurisprudencia del TSI se caracteriza por ser activa en la interpretación de las Leyes Básicas, aunque no se puede decir lo mismo acerca de la revisión judicial de la legislación israelí, por su escasa intervención en lo relacionado con los derechos fundamentales. Esto se refleja en sus decisiones, en especial los casos referentes a la seguridad nacional y que entra a ser un paradigma, desplazando cualquier intento de protección de los derechos fundamentales de la población palestina, como justificación de la amenaza contra el Estado israelí. A partir de este principio surge un concepto utilizado por el TSI como es el de la proporcionalidad. A continuación se analizará cuál es la teoría respecto a necesidad del Estado de preservar la seguridad, y cómo se puede hacer un balance entre las necesidades de la población palestina y la protección de la seguridad nacional. 


\section{Discrecionalidad Judicial y Legitimidad Constitucional}

ElTSI ha reforzado su papel dentro del Estado como defensor de los derechos humanos e intérprete constitucional frente a las acciones gubernamentales. No obstante, respecto a la política seguida por el gobierno dentro de los TO, no logra tener la fuerza suficiente para frenar las violaciones de los derechos humanos realizadas por el gobierno militar. Así las cosas, la doctrina que sigue es el reflejo de la moral como una forma de proteger los intereses de la población israelí frente a las amenazas a la seguridad. El argumento de la moral justifica en ocasiones la necesidad de causar un mal a la población ocupada con el fin de evitar un mal mayor a la sociedad israelí presente en los TO. Ahora bien, no es fácil adecuar esta doctrina a la ley porque no existe la certeza de saber en qué momento una acción dañina deba ser justificada como necesaria. Este concepto de daño menor no tiene ninguna validez razonable y por el contrario carece de argumentos válidos, aunque existen diferentes teorías morales al respecto (SEGEV, 2005-2006, p. 631).

En este sentido, Dworkin establece que la duda de los jueces no es sobre las disposiciones del derecho, sino la manera en la que ejercerán esa discreción judicial, cómo deben decidir respecto a un caso determinado y si es la mejor manera de hacerlo. El autor argumenta que en realidad lo que se presenta en estos casos es "una ambigüedad en el concepto de discreción" (DWORKIN, 2012, p. 131). Si los jueces tienen que decidir de acuerdo a una norma social que lo exija, no hay duda que la decisión debe ser tomada por un Tribunal Supremo, pero no porque estén en desacuerdo sobre lo que exige el derecho, sino porque no saben la forma en que deben hacer uso de esa discreción (DWORKIN, 2012, p. 131). En el conflicto palestino, el TSI no logra reforzar su posición y legitimidad como defensor de los derechos humanos porque no tiene una jurisprudencia coherente, es decir, estas decisiones no son guiadas siempre por una norma en concreto, sino que son generalmente resueltas por una interpretación de lo menos malo, o lo necesario para la defensa de la seguridad de la población israelí, en la lucha contra las amenazas terroristas.

El autor Segev (2005-2006, p. 632), manifiesta que las acciones ejecutivas y de los Tribunales deben guiarse por reglas que establezcan las normas, para evitar la posible discrecionalidad arbitraria de los funcionarios ejecutivos. Las reglas motivan un pensamiento racional y permiten alcanzar soluciones más justas y razonables. En un caso difícil las decisiones jurídicas de los Tribunales resuelven un conflicto y justifican la resolución del caso, sin embargo, esas decisiones no siempre tienen una justificación válida para el mismo. Esto es lo que ha sido llamado la doctrina de la justificación legalista (LYONS, 1998, p. 168).

ElTSI ha contribuido al desarrollo del Estado israelí a nivel social, no es una institución política y es visto por algunos sectores sociales como una institución imparcial porque investiga todo tipo de casos sin ninguna discriminación, dictando en ocasiones sentencias que van en contra de las resoluciones del gobierno militar en lo TO 
(BARZILAI, 1999, p. 16; 21-25). El TSI en una sentencia destacó: "The Supreme Court is the competent interpreter of the language of the law, as well as its condition at any given time" la función que desempeña dentro del sistema constitucional israelí sirve como una herramienta para hacer cumplir la voluntad del legislador, como representante legítimo de la sociedad. ${ }^{21}$

No obstante, su posición frente a los casos de los TO no deja de ser sesgada, porque solo decide la legalidad o ilegalidad de las acciones militares pero no cuestiona en ningún momento la situación de ocupación territorial en Palestina. En este sentido, Barzilai sostiene que la realidad de los jueces del TSI es diferente porque “... they often side with government policies, and, second, incumbent justices have only seldom and very reluctantly supported nominations of potential dissenting justices" (BARZILAI, 1999, p. 28). Esta visión es el reflejo de algunos grupos políticos nacionales e internacionales, porque argumentan que la función principal del TSI ha sido la de justificar las acciones gubernamentales bajo las disposiciones internacionales (KRETZMER, 1996, p. 95).

La actividad del TSI ha sido distinta, no se puede decir que sea coherente y constante pero en ocasiones sí ha ido en contra de las políticas gubernamentales con el objetivo de buscar la justicia social en los TO (SHAMIR, 1990, p. 795). La jurisprudencia ha contribuido para el desarrollo y empoderamiento constitucional en Israel reforzando el sistema democrático del Estado, proporcionando una administración más equitativa del gobierno (SHETREET, 2001, p. 240). La legitimación judicial concedida al TSI dentro de los TO ha sido criticada por considerarse que las reglas del derecho aplicadas son autoritarias y para nada se corresponde con la revisión judicial. Se puede decir que sus sentencias en general han creado una cultura de legitimación de la ocupación en Palestina, justificado siempre desde el paradigma de la seguridad nacional, pero no procura avances significativos en la retirada de los territorios, ni en el reconocimiento del derecho del pueblo palestino a tener un Estado (MEYDANI, 2006, p. 50).

ElTSI ha desarrollado dos conceptos que responden a la justificación de sus decisiones: "public consensus", "public morale" (BARZILAI, 1999, p. 20). Estos conceptos se desarrollan partiendo de los problemas que son sensibles para la población israelí y de interés para el gobierno militar, como punto central la moral y el deber ser, reconocido desde la necesidad del pueblo judío y sus valores. Por ello, hace un análisis del principio de proporcionalidad de las acciones de las FDI sin establecer una crítica sobre los procedimientos empleados. En términos generales, el TSI goza de legitimidad judicial por parte de la población israelí como palestina, aunque no estén de acuerdo con la efectividad en la protección de los derechos humanos en los TO, generando cuestionamientos entorno a su actividad (KRETZMER, 1996, p. 95).

Algunos miembros del gobierno y políticos piensan que la intervención del TSI en las decisiones sobre la seguridad ha dañado la efectividad de las acciones tomadas 
contra la violencia y el terror. En la práctica, poca ha sido el avance delTSI en la protección de los derechos fundamentales de la población palestina porque la mayoría de decisiones termina aceptando la discrecionalidad administrativa de las FDI en el uso de sus poderes en materia de seguridad. De manera que no pierden autonomía ni muchos menos se obstaculiza las decisiones tomadas por las el gobierno militar en aras de la protección general (KRETZMER, 1996, p. 96).

La función desempeñada por TSI no ha sido fácil porque se ha enfrentado no solo con la política del Estado frente a la ocupación y el predominio de la seguridad, sino también a los grupos religiosos. A su vez, con el carácter judío del Estado que tiene implícitos unos valores religiosos como esencia del mismo, lo cual hacen difícil su conciliación aunque han permitido reafirmar su posición frente a las ramas del poder público (SHETREET, 2001, p. 241).

\section{CONCLUSIÓN}

El TSI ha tenido un papel preponderante en el desarrollo del derecho Constitucional israelí, porque en la interpretación ha construido elementos que sirven para la concreción de los principios y los llamado valores dentro del Estado, que son el reflejo de los derechos fundamentales aceptados culturalmente, generando una creación o reinterpretación de normas, integración de las disposiciones normativas; favoreciendo la protección y cumplimiento de los derechos humanos en ausencia de una Carta Constitucional rectora.

El activismo judicial puede suponer una dificultad hermenéutica porque no existen unos parámetros claros respecto a una decisión activa, innovadora, de manera que el concepto de activo puede llegar a ser cambiante precisamente por la actividad interpretativa que no permite a los tribunales tener unos argumentos definidos. Por consiguiente, estos pueden cambiar dependiendo de la situación o los intereses que estén de por medio. Este activismo no tiene una ideología y se puede ejercer tanto de manera conservadora como liberal, en el caso de Israel, ceñida a los parámetros establecidos dentro de la Leyes Básicas, las cuales son parte de la cultura y el reconocimiento del pueblo judío. Esto a simple vista no sería algo malo porque reivindica principios éticos, morales y sociales, aceptados internacionalmente y que hacen parte de la doctrina internacional, sin embargo, sí choca con los intereses del pueblo palestino bajo ocupación, porque pertenecen a un grupo étnico distinto que reivindica otros principios.

Es precisamente esa interpretación del TSI que genera disensos en torno a lo que respecta la ocupación, porque no hace cambios frente a la opresión y violación de los derechos humanos, sino que interpreta las normas constitucionales e internacionales como método para justificar los actos del gobierno militar. Esto es solo un método subsidiario que da respuesta pronta a los conflictos presentes en la región, es decir, 
creando una indeterminación jurídica. Para esto ha hecho uso de la moral establecida en las Leyes Básicas del pueblo de Israel, creando un reconocimiento de las normas que aplica, una adecuación con la situación que se presente, intentando proporcionar una verdadera razonabilidad normativa, pero sin resolver el problema entre lo que disponen en esencia las Leyes Básicas y por otro lado, los principios del derecho internacional.

En algunos casos, el TSI ha ido más allá de la moral establecida en las Leyes Básicas, cambiando de manera arbitraria esa moral y los valores de la misma sociedad israelí, en aras de proteger los intereses de Israel en perjuicio de la población de los TO. Estas decisiones delTSI pueden generar un efecto contrario al de la búsqueda de la justicia y la igualdad dentro de los TO, porque no se sabe cuándo una interpretación de la norma pueda resultar ilegal pero necesaria para los intereses del Estado de Israel. Entonces, cuando hace esto, sus decisiones son contrarias y arbitrarias con las normas del derecho internacional.

La función del TSI frente a la ocupación ha sido la de hacer una interpretación de los valores constitucionales del Estado Israelí, frente a las disposiciones internacionales que obligan al Estado en situaciones de ocupación militar. Éste asignó al gobierno militar las funciones de gestor pero no reconoce la acción del mismo como ocupante, eliminando pues la ilegalidad de la ocupación y dejando a las autoridades militares de Israel la capacidad para equilibrar los intereses de las personas de una manera proporcional. Ha equiparado las autoridades ocupantes con los ocupados, lo cual genera una indefensión y una distorsión de la realidad que no permite a la población palestina pronunciarse por lo real, por lo que debe ser, la lucha por su soberanía y el rechazo de la misma por parte de Israel.

NOTAS

* Este artículo hace parte de la investigación adelantada en la Universidad San Buenaventura de Cali: un análisis hermenéutico - empírico de las condiciones para el conocimiento, interpretación y aplicación del derecho constitucional frente al derecho internacional— análisis de caso del Estado de Israel.

1 La creación de una Constitución para Israel fue decidida desde el Plan de Partición el 29 de noviembre de 1947, en la Asamblea General de las Naciones Unidas, después de la presentación del informe de la Comité Especial sobre Palestina (UNSCOP) que aprobó la Resolución 181(II) o Plan de Partición. Donde se hace la recomendación de partición de Palestina en Estado árabe, un Estado judío, en unión económica y con la ciudad de Jerusalén bajo mandato 
internacional. En esta Resolución 181 de las Naciones Unidas se estipula la adopción de una Constitución escrita para Israel y Palestina.

2 Las Leyes Básicas de Israel fueron promulgadas como una Constitución por capítulos, debido a las dificultades de alcanzar un consenso para crear una Constitución en el Estado de Israel. Para consultar las Leyes Básicas promulgadas hasta la fecha ver: <http://www.knesset.gov.il/description/eng/eng_mimshal_yesod1.htm> (consulta en: 20 de nov. de 2014).

3 Basic Law: Human Dignity and Liberty (1992).

4 Basic Law: Freedom of Occupation (1992) y modificada en 1994.

5 Cuando hablamos de los Territorios Ocupados por Israel en 1967, se hace referencia a: Cisjordania y Jerusalén Este, donde se han realizado cambios políticos, económicos, militares y gubernamentales, por parte del gobierno militar establecido en la región ocupada por Israel. En este caso, no se tendrá en cuenta el territorio de Gaza como ocupado, porque fue entregado por Israel de manera unilateral en el año 2005, por el entonces Primer Ministro Ariel Sharon. Con lo cual, hay una indeterminación jurídica sobre su situación actual, sí es un territorio ocupado o independiente.

6 Véase, Basic Law: Judiciary, párr.15 (a). Disponible en Web: <http://www.mfa.gov.il/mfa/aboutisrael/ state/law/pages/basic\%20laws\%20of\%20the\%20state\%20of\%20israel.aspx> (consulta: 20 de nov. de 2014). En el caso Ressler, el TSI establece su competencia para conocer de aquellas cuestiones donde un organismo público incumple con la ley. Ver, HCJ 910/86, Major (Res) Yehuda Ressler v. Minister of Defense, p. 2. Disponible en Web: <www.court.gov.il> (consulta en: 20 de nov. de 2014).

7 Es importante destacar que hay diversas opiniones en contra de la creación de un Tribunal Constitucional en Israel, una de ella es la del Juez Barak, quien lo ha manifestado tanto por escrito como públicamente. Propuesta que goza de apoyo del Knesset (Parlamento Israelí), aunque hasta la fecha no ha presentado ningún proyecto de ley para cambiar las competencias del TSI.

8 Veáse HCJ 2722/92, Mohammed Alamarin v. IDF Comander in Gaza Strip. Disponible en Web: <www.court.gov.il> (consulta: 20 de nov. de 2014). (en este caso, el TSI no permitió al gobierno militar la destrucción de un edificio entero en territorio palestino al considerar que no era una medida razonable).

9 Ver, Basic Law: The Judiciary (28-02-1984). Disponible en Web: <http://www.mfa.gov.il/mfa/aboutisrael/ state/law/pages/basic\%20laws\%20of\%20the\%20state\%20of\%20israel.aspx> (consulta en: 20 de nov. de 2014).

10 En este caso se puede consultar las siguientes sentencias del TSI donde aplica el derecho internacional y el israelí, para dar respuesta a la violación de los derechos de la población palestina por parte del gobierno militar: HCJ 8276/05, Adalah Legal Centre for Arab Minority Rights in Israel and others v. Minister of Defense. HCJ 1890/03, Bethehem Municipality v. Ministry of Defense. HCJ 5591/02, Yassin v. Commander of Kziot Military Párr. 8. HCJ 2150/07, Abu safiya v. Minister of Defense, párr. 33.

11 La Cueva Majpelá es un sitio sagrado tanto para judíos como musulmanes, se encuentra ubicada en Cisjordania y se cree que es el sitio de entierro de Abraham y Sara, Isaac y Rebeca, Jacob y Lea. A través de los años, en este lugar se han presentado actos de violencia por parte de miembros de una religión contra otra, causando víctimas. Los sábados y festivos los judíos pasan caminando cerca de la ciudad de Kiryat Arba a la Cueva de Majpelá, porque el uso de los vehículos está prohibida por el judaísmo en estos días. Van a la cueva a través de la ruta de peregrinación, que es un paso estrecho y por tanto, no es amplio para el paso de vehículos de seguridad o de rescate en el caso de un ataque terrorista.

12 Esta ciudad es un asentamiento israelí localizado en Cisjordania.

13 HCJ 10497/02, Hass v. IDF Commander inWest Bank, párr. 3. Disponible en Web: <www.court.gov.il> (consulta en: 20 de nov. de 2014).

14 Ibídem., párr. 3.

15 Ibídem., párr. 15. 
16 Ibídem, párr. 17.

17 La legislación citada fue: Basic Law: Human Dignity and Liberty; Palestine Order in Council, 1922, art. 83. Protection of Holy Places Law, 1967.

18 En la jurisprudencia del TSI se trataron distintos temas relacionados con los actos de los militares en los TO como: seguridad nacional, proporcionalidad, el uso de la tortura durante los interrogatorios, las detenciones administrativas, etc. En algunos casos se pronunció en contra y en otras donde consideró que no podía entrar a dirimir asuntos de competencia y conocimiento exclusivamente militar.

19 HCJ 910/86, Major (Res.) Yehuda Ressler v. Minister of Defense, p. 22. Disponible en Web: <www.court.gov.il> (consulta en: 20 de nov. de 2014).

20 Ver las siguientes sentencias: HCJ 680/88, Meir Chnitzer v. The Chief Military Censor. Disponible en Web: <www.court.gov.il> (consulta en: 26 de mar. de 2011); HCJ 393/82, Jamait Askan et al. v. IDF Commander of Judea and Samaria .

21 HCJ 6821/93, Bank Mizrahi v. Migdal Cooperative Village, p. 97.

\section{REFERENCIAS}

AGAMBEN, Giorgio. Homo sacer: sovereign power and bare life. California: Stanford University Press, 1998. ALBERT, Jeffrey M. Constitutional Adjudication without a Constitution: the case of Israel. Harvad Law Review. v. 82,1969 .

ALEXY, Robert. Teoría de la argumentación jurídica: la teoría del discurso racional como teoría de la fundamentación jurídica. Traducción de Manuel Atienza e Isabel Espejo. 2. ed. Madrid: Centro de Estudios Políticos y Constitucionales, 2008.

BARAK, Aharon. The Supreme Court as a Constitutional Court. Mishpat Uminshal. v. 6, 2003 (en hebreo). Un Juez reflexiona sobre su labor: el papel de un Tribunal Constitucional en una Democracia. Traducción de Estefanía Vela Barba. México: Suprema Corte Justicia de la Nación, 2008.

BARAK-EREZ, Daphne. From an unwritten to written constitution: The Israeli challenge in American perspective. Columbia Human Rights Law Review. v. 26, 1994-1995.

BARZILAI, Gad. Courts as Hegemonic Institutions: The Israeli Supreme Court in a Comparative Perspective. Israel Affairs. v. 5, n. 2, 1999.

BRENNAN JR., William J. The Quest to Develop a Jurisprudence of Civil Liberties in Times of Security Crises. Israel Yearbook on Human Rights. v. 18, 1988.

BRUCE PEABODY, Thomas H; WELLS Jr.; H. The politics of judicial independence: Courts, Politics, and the Public. Baltimore: JHU Press, 2010.

COHEN, Esther Rosalind. ¿Justice for Occupied Territorie? The Israeli High Court of Justice Paradigm. Columbia Journal of Transnatinonal Law. v. 24, 1985-1986.

COTRAN, Eugene; MALLAT, Chibli (edts.). The Arab-Israeli accords: Legal perspectives. London: Kluwer Law International, 1996

Human Rights. Israel Affairs. v. 11, n. 1, January 2005

DÍAZ REVORIO, Francisco Javier. Valores superiores e interpretación constitucional. Madrid: Centro de Estudios Políticos y Constitucionales, 1997.

DOTAN, Yoav. Judicial Rhetoric, Government, Lawyers, and Human Rights: The Case of the Israeli High Court of Justice during Intifada. Law \& Society Review. v. 33, 1999.

. Judicial Accountability: The High Court of Justice and the Phenomenon of Judicial Hyperactivism. Israel Affairs, summer, v. 8, n. 4, 2002.

. Legalising the Unlegaliseable: Terrorism, Secret Services and Judicial Review in Israel 1970-2001.

HERTOGH, Marc; HALLIDAY, Simon. Judicial Review and Bureaucratic Impact: International and interdisciplinary Perspectives. Cambridge, Cambridge University Press, 2004. 
DWORKIN, Ronald. El imperio de la justicia. Barcelona: Gedisa, 2005. . Los derechos en serio. Barcelona: Ariel, 2012.

EDELMAN, Martin. Courts, Politics, and Culture in Israel. Charlottesville: University Press of Virginia, 1994.

GAVISON, Ruth. A Constitution for Israel: Lessons from the American Experiment. Azure. v. 12, 2002.

The Israeli Constitutional Process: Legislative Ambivalence and Judicial Resolute Drive. The Hebrew University of Jerusalem. Paper n. 380, 2005.

Legislatures and the Phases and Components of Constitutionalism. In: BAUMAN, Richard KAHANA, Tsvi.

The least examined branch: The Role of Legislatures in the Constitutional State. Cambridge: Cambridge University Press, 2006.

GOLDSTEIN, Stephen. The Protection of Human Rights by Judges: The Israeli Experience. In: GIBNEY, Mark; FRANKOWSKI, Stanislaw. Judicial Protection of Human Rights: myth or reality? Westport: Greenwood Publising Group, 1999.

GOMES CANOTILHO, José Joaquim. Teoría de la Constitución. Madrid: Dykinson, 2004.

GORDON, Evelyn. Is It Legitimate to Critize the Supreme Court? Azure. v. 3, 1998. Disponible en:

<http://www.azure.org.il/article.php?id=397>. Consulta en: 20 de nov. de 2014.

HABA, Enrique P. Rehabilitación del no-saber en la actual Teoría del Derecho. El Bluff Dworkin (Retorno al realismo ingenuo y apogeo del efecto-Vicente, lanzados por una reencarnación más del Prof. Beale). DOXA, n. 24, 1989.

HÄBERLE, Peter. El Estado Constitucional. México: Universidad Nacional Autónoma de México, 2003.

HART, Herbert Lionel A. The Concept of Law. 3. ed. Oxford: Clarendon Law, 2012.

HERTOGH, Marc; HALLIDAY, Simon. Judicial Review and Bureaucratic Impact: International and interdisciplinary Perspectives. Cambridge: Cambridge University Press, 2004.

HOLZMAN-GAZIT, Yifat. Land Expropriation in Israel: Law, Culture and Society. England: Ashgate, 2007.

HONGJU KOH, Harold. The Spirit of the Laws. Harvard International Law Journal. v. 43, n. 1, 2002.

KOSKENNIEMI, Martti. ¿Occupied zone - A zone of reasonableness? Israel Law Review. v. 41, 2008.

KRETZMER, David. Domestic Politics, Law and the Peace Process: A View from Israel. In:

LYONS, David. Aspectos morales de la teoría jurídica. Ensayos sobre la ley, la justicia y la responsabilidad política.

Barcelona: Gedisa, 1998.

MCEWEN, Craig A.; MAIMAN, Richard J. In search of Legitimacy: Toward an Empirical Analysis. Law \& Policy. v. 8, n. 3, July 1986.

MEYDANI, Assaf; MIZRAHI, Shlomo. The Politics and Strategies of Defending Human Rights: The Israeli Case. Israel Law Review. v. 39, 2006.

MORESO, Juan José. La indeterminación del derecho y la interpretación de la Constitución. Madrid: Centro de Estudios Políticos y Constitucionales, 1997.

NAVOT, Suzie. The Constitutional Law in Israel. Netherlands: Kluwer Law International, 2007.

NETANYAHU, Shoshana. The Supreme Court of Israel: A Safeguard of the Rule of Law. Pace International Law Review. v. 5, n. 1, 1993 .

POZZOLO, Susanna. Neoconstitucionalismo y especificidad de la interpretación constitucional. DOXA. v. 21, n. II, 1998. ROBERTS, Adam. Prolonged military occupation. The Israeli-occupied territories since 1967. American Journal International Law. v. 84, 1990.

SCHAUER, Frederick. The occasions of constitutional interpretation. Boston University Law Review. v. 72, 1992.

SEGAL, Zeev. A Constitution without Constitution:The Israeli Experience and the American Impact. Capital University Law. v. 21, 1992.

SEGEV, Re'em. Moral justification, administrative power and emergencies. v. 53. Cleveland State Law Review. 2005-2006. SHAMIR, Ronen. Landmark Cases and the Reproduction of the Legitimacy: The case of Israel's Highs Courts of Justice. Law and Society Review. v. 24, 1990.

SHAPIRO, Scott J. The "Hart-Dworkin" debate: a short guide for the perplexed. Michigan Law School. n. 77, March 2007. SHETREET, Shimon. Developments in Constitutional Law: Selected Topics. Israel Law Review. v. 24, 1990. . The Critical Challenge of Judicial Independence in Israel. In: RUSSELL, Peter H. O'BRIEN, David, M. (edits.). Judicial Independence in the Age of Democracy: critical perspectives from around the world. United States of America: The University Press of Virginia, 2001. Resolving the Controversy over the Form and Legitimacy of constitutional Adjudication in Israel:A Blueprint for Redefining the Role of the Supreme Court and the Knesset. Tulane Law Review. v. 77, 2002-2003. STRASCHNOV, Amnon. The Judicial System in Israel. Tulsa Law Journal. v. 34, 1998-1999.

SENTENCIAS TRIBUNAL SUPREMO DE ISRAEL

HCJ 393/82, Jamait Askan et al.v. IDF Commander of Judea and Samaria.

HCJ 910/86, Major (Res.) Yehuda Ressler v. Minister of Defense. 
HCJ 680/88, Meir Chnitzer v. The Chief Military Censor.

HCJ 2722/92, Mohammed Alamarin v. IDF Comander in Gaza Strip.

HCJ 6821/93, Bank Mizrahi v. Migdal Cooperative Village.

HCJ 10497/02, Hass v. IDF Commander in West Bank.

HCJ 5591/02, Yassin v. Commander of Kziot Military.

HCJ 1890/03, Bethehem Municipality v. Ministry of Defense.

HCJ 8276/05, Adalah Legal Centre for Arab Minority Rights in Israel and others v. Minister of Defense.

HCJ 2150/07, Abu safiya v. Minister of Defense.

Yaniza Giraldo Restrepo

ygiraldo1ausbcali.edu.co |

DOCTORA EN DERECHO POR LA UNIVERSIDAD

CARLOS III DE MADRID

MÁSTER EN DERECHO PÚBLICO POR LA UNIVERSIDAD

CARLOS III DE MADRID

MÁsTER EN DERECHO INTERNACIONAL Y RELACIONES INTERNACIONALES DE LA UnIVERSIDAD COMPLUTENSE DE MADRID

DoCENTE DE TIEMPO COMPLETO DE LA UNIVERSIDAD

de San Buenaventura - Cali

PROFESORA DE DERECHO INTERNACIONAL PÚBLICO 
